\section{Painful Mass of the Neck coming down to Diagnosis of Takayasu's Arteritis: A Case Report}

\author{
Bahar Kayahan Sirkeci* \\ Department of Head and Neck Surgery, LIV Hospital Ear Nose Throat Dis-
} ease, Yuksek Ihtisas University Medical Faculty, Ankara, Turkey

\begin{abstract}
Takayasu's Arteritis, is a rare, chronic idiopathic vasculitis which affects the large vessels in the body and the patients are usually young women. The aorta and its main branches are the primary vessels affected, with the most typical features reflected as ischemia or aneurysm formation. With Takayasu'sArteritis being a rare condition and its acute phase presentation often similar to, diagnosis is often difficult.
\end{abstract}

Here is presented a case report of a young woman with Takayas's Arteritis, who presented a painful, tender mass localized anterior aspect of sternocleido mastoid muscle. The patient was investigated with a number of imaging modalities and diagnosed with Takayasu's Arteritis, involving left common and internal carotid arteries. She was treated as per protocol for Takayasu's Arteritis.

This case report highlights that a differential diagnosis should never be dispelled based upon a "typical" history. The importance of modern day imaging techniques such as CT and MRI can often be paramount to confirming a diagnosis and the extent of the pathology.

Keywords: Carotidynia; Inflammatory disease; Lymphadenopathy; Takayasu's arteritis

\section{Introduction}

Takayasu's Arteritis (TA) is a chronic idiopathic vasculitis that variably involves the aorta and/or its main branches and the coronary

*Corresponding author: Bahar Kayahan Sirkeci, Department of Head and Neck Surgery, LIV Hospital Ear Nose Throat Disease, Yuksek Ihtisas University Medical Faculty, Ankara, Turkey, Tel: +90 5336576826; E-mail: baharkayahan@ hotmail.com

Citation: Sirkeci BK (2020) Painful Mass of the Neck coming down to Diagnosis of Takayasu's Arteritis: A Case Report. J Otolaryng Head Neck Surg 6: 43.

Received: April 21, 2020; Accepted: May 27, 2020; Published: June 03, 2020

Copyright: (C) 2020 Sirkeci BK. This is an open-access article distributed under the terms of the Creative Commons Attribution License, which permits unrestricted use, distribution, and reproduction in any medium, provided the original author and source are credited. and pulmonary arteries in 50-80\%. Inflammation results in stenosis, occlusion or aneurysm formation [1-3]. Aneurysms may rarely progress to vascular rupture and death. TA is a difficult disease to deal with. First, early diagnosis is difficult and requires clinical awareness and suspicion. Second, and even more important, is the lack of standard and reliable parameters reflecting disease activity [4].

The incidence of TA is about 2/10,000 person-years, with tenfold predominance in women, especially under age of 40 . There is a marked ethnic preference, with high prevalence rates in Asian countries, while less common in Caucasian populations $[1,2]$.

Here is reported a case of TA which presented with symptoms and signs of painful neck fullness, and got the TA diagnose with neck U1trasound (US), Computerized Tomography (CT) and Magnetic Resonance Imaging (MRI).This case report highlights the importance of physical examination, clinic suspicion, differential diagnoses and the importance of modern day imaging in the diagnosis.

\section{Case Presentation}

A 38-year old female presented to our clinic with a history of left submandibular tenderness, pain with neck movement and dullness. The pain was of sudden onset, started two days ago. The pain was increasing especially elevating the head while moving it toward the contralateral side. She first appealed to her dentist to check if there was a problem regarding teeth, after examination the dentist consulted the patient our clinic in case of sialoadenitis or lymph adenopathy. During anamnesis the patient gave the information that she had leftsided tinnitus for a week ago and sometimes she felt her heartbeat in her left ear. Her physical examination was in normal ENT examination except for solid tenderness and fullness between left submandibular gland and anterior border of left sternocleidomastoid muscle. There was not any lymph node or well-bordered mass in this region. There is not any systemic disease or regular medication intake, in her family history there was hypertension only.

A neck ultrasound was carried out to investigate differential diagnoses. An increased wall thickness from the left carotid bulbus to left internal carotid artery was detected, causing a suspicion of dissection. And the pain was named as "carotidynia" from that moment. She immediately went under CT angiography, there was not any leakage through the carotid system but wall thickening, vertically $3 \mathrm{~cm}$, on the left carotid bulbus and distal of the left common carotid artery was detected, also there was contour irregularity on the left internal carotid artery origin which was accepted as an intra mural hematoma or inflammatory vasculitis process (Figure 1).

The patient was consulted to rheumatology department, her blood tests were taken; Erythrocyte Sedimentation Rate (ESR) was 30mm, serum amyloid protein was $0.76 \mathrm{mg} / \mathrm{dL}$; ENA panel, Anti dsDNA, Anticardiolipin antibody IgG and IgM, AntiBeta-2 antibody IgM and IgG, Anti CCP, ANA, p-ANCA and Lupus anticoagulant were negative, C-reactive Protein (CRP) and Rheumatoid Factor (RF) levels was in normal range. It was especially noted that pulse was weaker 
on the left arm. As an initial diagnosis of TA, neck MRI with intravenous contrast was carried out and vasculitis process was confirmed involving distal part of left common carotid artery, carotid bifurcation and proximal internal carotid artery wall (Figures 2 and 3). Diagnosed with TA, the medical treatment is scheduled as; methyl prednisolon $1 \mathrm{~g}$ once-a-day (OD) for three days followed by prednisolone $1 \mathrm{mg}$ / $\mathrm{kg}(60 \mathrm{mg})$ for two weeks was commenced, along with my cophenolatemofetil $500 \mathrm{mg}$ per oral (PO) bis in die (BD), acetyl salicyclic acid 100mg PO OD, Enoxaparine sodium 6000 anti-Xa IU-0.6 ml. These were well tolerated with no side effects experienced. After two weeks of treatment patient was evaluated on control appointment by both ENT and the rheumatology department. Fullness was still going on but tenderness almost diminished. Prednisolone dosage was decreased to 30mg PO OD / 4 weeks, $20 \mathrm{mg}$ PO OD /4 weeks, 10mg/ 4 weeks and then stopped; and mycophenolate mofetil was increased to $750 \mathrm{mg}$ PO BD. It has been 10 months since the patient presented and regular follow-ups continue at increasing intervals from TA perspective, she remains without any complaints.

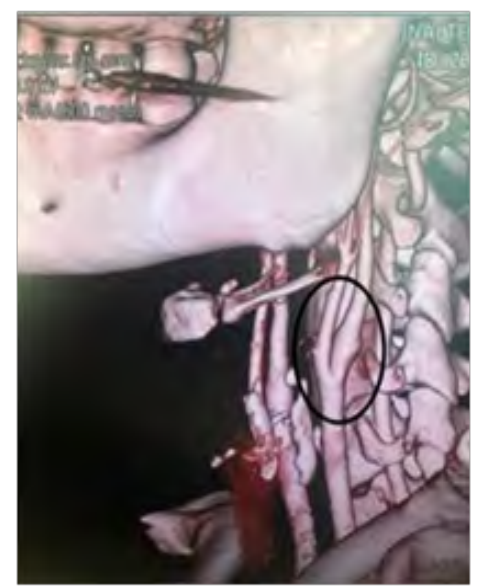

Figure 1: The wall thickening, on the left carotid bulbus and distal of the left common carotid artery, CT angiography.
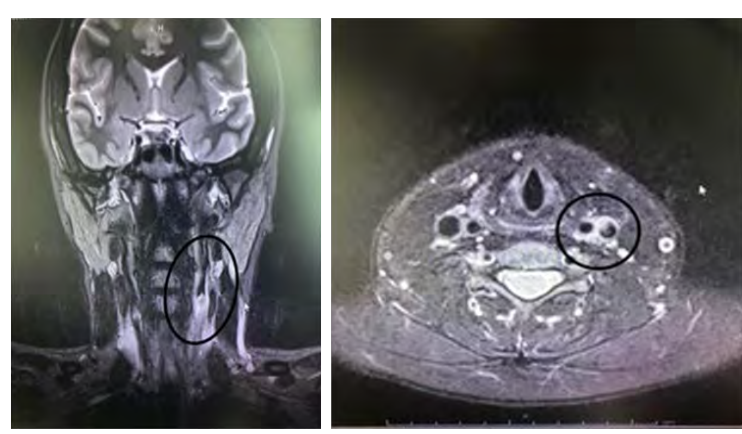

Figures 2 and 3: Vasculitis process involving distal part of left common carotid artery, carotid bifurcation and proximal internal carotid artery wall.

\section{Discussion}

TA is a rare, large-vessel arteritis of unknown etiology. It is a life-threatening disease without intensive treatment [1-3]. The 5-year survival is $92.9 \%$ and 10 -year survival is $87.2 \%$ [2]. Histologically, it is characterized by a granulomatous infiltrative process of the arterial wall (acute stage) with marked intimal proliferation and fibrosis of the media and adventitia (fibrotic stage), resulting in severe stenosis, occlusion or aneurysmal dilation (from extensive destruction of elastic fibers in the media) $[4,5]$.

The clinical manifestations of the disease are divided into early and late phases. The early or pre-pulse less phase is often characterized by non-specific signs and general symptoms of systemic inflammation (fever, weight loss, night sweats, weakness, myalgia, arthralgia), whereas the late or pulse less phase (consequences of vascular inflammation or fibrosis) may be associated with symptoms of ischemia of limb, renovascular hypertension, pulmonary hypertension, pulmonary embolism, myocardial ischemia and neurological symptoms [3-6].

The term carotidynia was defined as "carotid pain by Fay in 1927, to describe the patients presenting with dull, throbbing pain directly over the carotid artery (either unilateral or bilateral) that was exacerbated with light pressure. In 1988, the International Headache Society (IHS) declared acute idiopathic carotidynia to be a separate entity using four clinical criteria for diagnosis; including appropriate investigations not revealing structural abnormality [7]. In 1994, the validity of the HIS classification of carotidynia was questioned by a review and the controversy led the HIS to remove carotidynia as a distinct entity, instead referring it as a syndrome of unilateral neck pain. Today, carotidynia remains as a controversial subject. Many authors continue to use the term to describe neck pain due to any etiology, whereas some maintain that it is a separate disease entity $[7,8]$.

Carotidynia is a neck pain syndrome associated with tenderness on palpation over the carotid bifurcation. The pain is mild to moderate intensity typically dull, throbbing and continuous just like in our case. The pain may be aggrevated by swallowing, coughing, sneezing or elevating the head while moving it toward the contralateral side. Carotidynia has been reported in $32 \%$ of patients with TA. Differential diagnosis for carotidynia includes large-vessel vasculitis such as TA and temporal arteritis, arteriosclerosis, thrombosis, dissection, aneurysm, pharyngitis, lymphadenitis, submandibular gland disease and thyroiditis [3].

Although conventional angiography has been the gold standard imaging tool for diagnosis and evaluation of TA, being invasive with procedural morbidity and mortality risks it is not so commonly used. The initial diagnosis of TA is made using serological markers, including CRP and ESR, combined with imaging modalities including US, $\mathrm{CT}$ angiography, MRI and FDG-PET if necessary. According to the recommendations for use of imaging in large-vessel vasculitis outlined by the European League Against Rheumatism (EULAR), MRI should be used as the first imaging test for diagnosis TA. MRI with enhancement of the thickened walls with gadolinium contrast suggests the active phase of TA. FDG-PET, CT and US as alternative imaging modalities in patients suspected TA [3,5]. During our diagnosis process; neck US was wanted to understand the etiology of neck pain, with the suspicion of US, CT angiography was carried out to understand if there was a leakage through the arterial wall. The contrasted MRI had a key role to name the disease TA.

Due to the rarity of TA and the lack of international collaborative studies, treatment modalities are proposed based on clinical observations and experts' opinions. Corticosteroids (CS) remain the main stay 
of medical treatment; however, TA is often resistant to treatment, so pulse therapy with methyl prednisolone should be considered. Several immunosuppressive agents such as metothrexate, cyclophosphamide, azathioprine, cyclosporine and mycophenolate mofetil have been used as steroid-sparing agents in TA patients with varying efficacy. Obstructive lesions need to be managed by revascularization techniques such as angioplasty and surgery $[3,6,9,10]$.

In conclusion, carotidynia is a painful symptom that may be underrated, but should be taken into consideration in case of non-palpable neck pain. It might be a clinical sign of Takayasu's arteritis and other large-vessel vasulitis. Non-invasive imaging modalities such as US, CT and MRI allow earlier diagnosis than conventional imaging. MRI enhanced with gadolinium and PET scan offer additional information regarding the staging, dynamic evaluation and management of Takayasu's arteritis

\section{Acknowledgements}

The author declares that informed consent was obtained from the patient referred on this report regarding the publication of all included information.

\section{Disclosure Statement}

The author hereby declares having no conflicts of interest to declare regarding the publication of this article.

\section{Funding Sources}

This research received no specific grant from any funding agency in the public, commercial, or not-for-profit sectors.

\section{Statement of Ethics}

This research involves the patient's informed consent that was given willingly. In addition to the patient's informed consent, the research was conducted ethically in accordance with the World Medical Association Declaration of Helsinki.

\section{References}

1. O'Connor MB, Murphy E, O'Donovan N, Murphy M, Phelan MJ, et al (2008) Takayasu's Arteritis presenting as a dissecting aortic aneurysm history: a case report. Cases J 1: 52.

2. Andrzejewska K, Starba A, Misterska-Skóra M, Wiland P, Guziński M (2017) Palpable mass of the neck in the course of Takayasu arteritis. Reumatologia 55: 48-52.

3. Zeina AR, Slobodin G, Barmeir E (2008) Takayasu's arteritis as a cause of carotidynia: clinical and imaging features. Isr Med Assoc J 10: 158-159.

4. Keser G, Direskeneli H, Aksu K (2014) Management of Takayasu arteritis: a systematic review. Rheumatology (Oxford) 53: 793-801.

5. Oguro E, Ohshima S, Kikuchi-Taura A, Murata A, Kuzuya K, et al. (2019) Diffusion-weighted Whole-body Imaging with Background Body Signal Suppression (DWIBS)as a Novel Imaging Modality for Disease Activity Assessment in Takayasu's Arteritis. Intern Med 58: 1355-1360.

6. Guo J, Zhang G, Tang D, Zhang J (2017) A case report of Takayasu arteritis with aortic dissection as initial presentation. Medicine (Baltimore) 96: e8610.

7. Elkins A, Barakate M, Henderson J, Grieve S (2015) A Pain in the Throat: A 19-year History of Symptoms Relating to the Carotid Artery. Wien Klin Wochenschr 127: 143-145.

8. Stanbro M, Gray B, Kellicut D (2011) Carotidynia: Revisiting an Unfamiliar Entity. Ann Vasc Surg 25: 1144-1153.

9. Watanabe Y, Miyata T, Tanemoto K (2015) Current Clinical Features of New Patients With Takayasu Arteritis Observed From Cross-Country Research in Japan: Age and Sex Specificity. Circulation 132: 1701-1709.

10. Nasser M, Cottin V (2018) The Respiratory System in Autoimmune Vascular Diseases. Respiration 96: 12-28. 


\section{dI}

Advances In Industrial Biotechnology | ISSN: 2639-5665

Advances In Microbiology Research | ISSN: 2689-694X

Archives Of Surgery And Surgical Education | ISSN: 2689-3126

Archives Of Urology

Archives Of Zoological Studies | ISSN: 2640-7779

Current Trends Medical And Biological Engineering

International Journal Of Case Reports And Therapeutic Studies | ISSN: 2689-310X

Journal Of Addiction \& Addictive Disorders | ISSN: 2578-7276

Journal Of Agronomy \& Agricultural Science | ISSN: 2689-8292

Journal Of AIDS Clinical Research \& STDs | ISSN: 2572-7370

Journal Of Alcoholism Drug Abuse \& Substance Dependence | ISSN: 2572-9594

Journal Of Allergy Disorders \& Therapy | ISSN: 2470-749X

Journal Of Alternative Complementary \& Integrative Medicine | ISSN: 2470-7562

Journal Of Alzheimers \& Neurodegenerative Diseases | ISSN: 2572-9608

Journal Of Anesthesia \& Clinical Care | ISSN: 2378-8879

Journal Of Angiology \& Vascular Surgery | ISSN: 2572-7397

Journal Of Animal Research \& Veterinary Science | ISSN: 2639-3751

Journal Of Aquaculture \& Fisheries | ISSN: 2576-5523

Journal Of Atmospheric \& Earth Sciences | ISSN: 2689-8780

Journal Of Biotech Research \& Biochemistry

Journal Of Brain \& Neuroscience Research

Journal Of Cancer Biology \& Treatment | ISSN: 2470-7546

Journal Of Cardiology Study \& Research | ISSN: 2640-768X

Journal Of Cell Biology \& Cell Metabolism | ISSN: 2381-1943

Journal Of Clinical Dermatology \& Therapy | ISSN: 2378-8771

Journal Of Clinical Immunology \& Immunotherapy | ISSN: 2378-8844

Journal Of Clinical Studies \& Medical Case Reports | ISSN: 2378-8801

Journal Of Community Medicine \& Public Health Care | ISSN: 2381-1978

Journal Of Cytology \& Tissue Biology | ISSN: 2378-9107

Journal Of Dairy Research \& Technology | ISSN: 2688-9315

Journal Of Dentistry Oral Health \& Cosmesis | ISSN: 2473-6783

Journal Of Diabetes \& Metabolic Disorders | ISSN: 2381-201X

Journal Of Emergency Medicine Trauma \& Surgical Care | ISSN: 2378-8798

Journal Of Environmental Science Current Research | ISSN: 2643-5020

Journal Of Food Science \& Nutrition | ISSN: 2470-1076

Journal Of Forensic Legal \& Investigative Sciences | ISSN: 2473-733X

Journal Of Gastroenterology \& Hepatology Research | ISSN: 2574-2566
Journal Of Genetics \& Genomic Sciences | ISSN: 2574-2485

Journal Of Gerontology \& Geriatric Medicine | ISSN: 2381-8662

Journal Of Hematology Blood Transfusion \& Disorders | ISSN: 2572-2999

Journal Of Hospice \& Palliative Medical Care

Journal Of Human Endocrinology | ISSN: 2572-9640

Journal Of Infectious \& Non Infectious Diseases | ISSN: 2381-8654

Journal Of Internal Medicine \& Primary Healthcare | ISSN: 2574-2493

Journal Of Light \& Laser Current Trends

Journal Of Medicine Study \& Research | ISSN: 2639-5657

Journal Of Modern Chemical Sciences

Journal Of Nanotechnology Nanomedicine \& Nanobiotechnology | ISSN: 2381-2044 Journal Of Neonatology \& Clinical Pediatrics | ISSN: 2378-878X

Journal Of Nephrology \& Renal Therapy | ISSN: 2473-7313

Journal Of Non Invasive Vascular Investigation | ISSN: 2572-7400

Journal Of Nuclear Medicine Radiology \& Radiation Therapy | ISSN: 2572-7419

Journal Of Obesity \& Weight Loss | ISSN: 2473-7372

Journal Of Ophthalmology \& Clinical Research | ISSN: 2378-8887

Journal Of Orthopedic Research \& Physiotherapy | ISSN: 2381-2052

Journal Of Otolaryngology Head \& Neck Surgery | ISSN: 2573-010X

Journal Of Pathology Clinical \& Medical Research

Journal Of Pharmacology Pharmaceutics \& Pharmacovigilance | ISSN: 2639-5649

Journal Of Physical Medicine Rehabilitation \& Disabilities | ISSN: 2381-8670

Journal Of Plant Science Current Research | ISSN: 2639-3743

Journal Of Practical \& Professional Nursing | ISSN: 2639-5681

Journal Of Protein Research \& Bioinformatics

Journal Of Psychiatry Depression \& Anxiety | ISSN: 2573-0150

Journal Of Pulmonary Medicine \& Respiratory Research | ISSN: 2573-0177

Journal Of Reproductive Medicine Gynaecology \& Obstetrics | ISSN: 2574-2574

Journal Of Stem Cells Research Development \& Therapy | ISSN: 2381-2060

Journal Of Surgery Current Trends \& Innovations | ISSN: 2578-7284

Journal Of Toxicology Current Research | ISSN: 2639-3735

Journal Of Translational Science And Research

Journal Of Vaccines Research \& Vaccination | ISSN: 2573-0193

Journal Of Virology \& Antivirals

Sports Medicine And Injury Care Journal | ISSN: 2689-8829

Trends In Anatomy \& Physiology | ISSN: 2640-7752

Submit Your Manuscript: http://www.heraldopenaccess.us/Online-Submission.php 